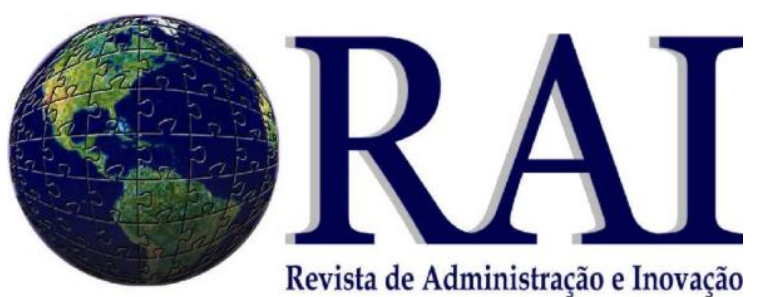

\title{
ARTIGOS
}

\section{INOVAÇÃO NO PROCESSO DE AVALIAÇÃO DO DESEMPENHO ORGÄNIZACIONAL: O USO DA DIMENSÃO INTEGRATIVA}

\section{Ademar Dutra}

Doutor em Engenharia da Produção pela Universidade do Sul de Santa Catarina - UNISUL Professor do Mestrado em Administração da Universidade do Sul de Santa Catarina - UNISUL E-mail: ademar.dutra@unisul.br [Brasil]

\section{Leonardo Ensslin}

Doutor em Engenharia Industrial e Sistemas - University of Southern California

Professor Titular da Universidade Federal de Santa Catarina - UNISUL

E-mail: ensslin@deps.ufsc.br [Brasil]

\section{Sandra Rolim Ensslin}

Doutora em Engenharia da Produção pela Universidade do Sul de Santa Catarina - UNISUL

Professora adjunto da Universidade Federal de Santa Catarina - UNISUL

E-mail: sensslin@mbox1.ufsc.br [Brasil]

\section{Marcus Vinícius Andrade Lima}

Doutor em Engenharia da Produção pela Universidade do Sul de Santa Catarina - UNISUL

Professor titular da Universidade do Sul de Santa Catarina - UNISUL

E-mail: vinicius@unisul.br [Brasil]

\section{Ana Lucia Miranda Lopes}

Doutora em Engenharia da Produção pela Universidade do Sul de Santa Catarina - UNISUL

Professora da Universidade Federal de Santa Catarina - UNISUL

E-mail: analopes@unisul.br [Brasil]

\section{Resumo}

O objetivo desse artigo é discutir a incorporação da dimensão integrativa no processo de avaliação de desempenho organizacional, tendo como base a Metodologia Multicritério de Apoio à Decisão Construtivista - MCDA-C. Esta dimensão busca identificar as variáveis de desempenho internas de cada subsistema organizacional, sobretudo, aquelas decorrentes das inter-relações e interconexões entre os diversos subsistemas, enfatizando a escolha das estratégias mais relevantes para a melhoria de desempenho do sistema como um todo. Como resultado, a incorporação da dimensão integrativa possibilita: (i) identificar as variáveis que um subsistema afeta e é afetado pelo desempenho de outro subsistema; (ii) permitir que cada subsistema, à medida em que se deparar com duas alternativas pontuais para aperfeiçoar o desempenho, opte pela alternativa que apresentar maior contribuição para o desempenho global do sistema; e, (iii) identificar as estratégias mais adequadas para melhorar o desempenho do sistema como um todo, permitindo um equilíbrio dinâmico e global.

Palavras-chave: Avaliação, avaliação de desempenho organizacional, dimensão integrative, MCDA-C.

RAI - Revista de Administração e Inovação ISSN: 1809-2039

Organização: Comitê Científico Interinstitucional

Editor Científico: Milton de Abreu Campanario

Avaliação: Double Blind Review pelo SEER/OJS

Revisão: gramatical, normativa e de formatação

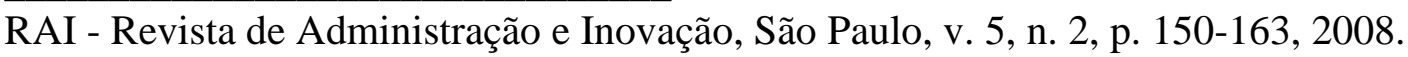


ARTIGOS - Inovação no processo de avaliação do desempenho organizacional: o uso da dimensão integrativa

\section{INTRODUÇÃO}

No contexto organizacional contemporâneo, a busca de competitividade tem exigido das organizações preocupações permanentes com sistemas de avaliação de seu próprio desempenho.

Neste cenário, o presente trabalho se fundamenta na inovação no processo de avaliação de desempenho organizacional calcada na Metodologia Multicritério de Apoio à Decisão Construtivista - MCDA-C (COSTA, 1995; COSTA; VINCKE, 1990; ENSSLIN; LIMA, 2008; ENSSLIN; MONTIBELLER NETO; NORONHA, 2001; KEENEY, 1992; LANDRY, 1995; ROY, 1993; ROY; VANDERPOOTEN, 1996), propondo a incorporação de uma nova dimensão à prática de avaliação de desempenho, a saber, a dimensão integrativa.

Com isso, a pergunta de pesquisa está estruturada da seguinte forma: Como incorporar a dimensão integrativa nos processos de avaliação de desempenho organizacional, tendo como base a Metodologia Multicritério de Apoio à Decisão Construtivista? Com vistas a responder à pergunta de pesquisa proposta pelos autores, o objetivo do presente trabalho - de caráter teórico conceitual (ALAVI; CARLSON, 1992) por meio de um método científico dedutivo (RICHARDSON, 1999) - é discutir a incorporação da dimensão integrativa no processo de avaliação de desempenho organizacional, tendo como base a Metodologia Multicritério de Apoio à Decisão Construtivista (MCDA-C).

Cumpre esclarecer a afiliação teórica do trabalho, no que tange o conceito de avaliação de desempenho organizacional. Os autores afiliam-se a definição de AD formulada por Igarashi et al. (2008). Segundo a ótica desses pesquisadores, uma avaliação deve investigar:

(i) o que vai ser avaliado - ou seja, conhecer o objeto da avaliação, incluindo aqui a sua identidade, a cultura sobre a qual esta identidade é construída, as instâncias que respondem pelo objeto a ser avaliado, resultando nos objetivos a serem perseguidos; (ii) como proceder à avaliação - ou seja, identificar como cada objetivo será avaliado e quanto cada objetivo contribui para a avaliação do todo, possibilitando a identificação do perfil de desempenho do objeto avaliado; (iii) como conduzir ao gerenciamento interno - com base na análise das fragilidades e potencialidades identificadas para sugerir ações de aperfeiçoamento promovendo a alavancagem do desempenho institucional (IGARASHI et al., 2008, p. 119).

A dimensão integrativa, no contento da avaliação de desempenho organizacional, busca identificar as variáveis de desempenho internas de cada subsistema e, sobretudo, as decorrentes das inter-relações e interconexões entre os diversos subsistemas, enfatizando a escolha das estratégias mais relevantes para a melhoria do sistema como um todo, possibilitando um equilíbrio dinâmico e global. O contexto de aplicação da dimensão é o ambiente organizacional, caracterizado por sistema e subsistemas, podendo, ainda, apresentar outros desdobramentos, de acordo com a estrutura da organização ou de seus processos de trabalho.

Esta pesquisa pretende oferecer uma contribuição para aprimorar modelos de avaliação de desempenho organizacional que atendam às demandas do contexto organizacional da atualidade.

\section{A METODOLOGIA MULTICRITÉRIO CONSTRUTIVISTA DE APOIO À DECISÃO - MCDA-C}

RAI - Revista de Administração e Inovação, São Paulo, v. 5, n. 2, p. 150-163, 2008. 
Ademar Dutra, Leonardo Ensslin, Sandra Rolim Ensslin, Marcus Vinícius Andrade Lima e Ana Lucia Miranda Lopes

A MCDA-C se constitui em uma ferramenta de apoio à tomada de decisão em um contexto multicritério, cujas premissas podem ser sumarizadas conforme segue: (a) consenso com relação ao fato de que, nos problemas decisórios, existem múltiplos critérios; (b) consenso com relação ao fato de que, em substituição à noção de melhor solução, propõem-se à busca por uma solução que melhor se enquadre nas necessidades do decisor e no contexto decisional como um todo.

Neste contexto, a partir dos pensamentos de Costa (1988, 1993), Costa e Vincke (1990), Roy (1993), destaca-se como característica da MCDA-C: (i) o reconhecimento dos limites da objetividade e a conseqüente aceitação da subjetividade; (ii) a incorporação da dimensão construtivista, segundo a qual ocorre aprendizagem constante e evolutiva do contexto decisório, em oposição à crença em um conjunto de ferramentas para permitir uma solução única e melhorada do problema (convicção do construtivismo calcada na noção de participação conduzindo a um novo paradigma de aprendizagem); (iii) a aceitação da inseparabilidade dos elementos de natureza objetiva e dos elementos de natureza subjetiva (convicção da interpenetração em consonância com o aspecto de indivisibilidade do sistema); (iv) a proposta de uma atividade de apoio à decisão, o diferencial central da MCDA-C frente a outras metodologias; (v) a presença deste apoio em todas as etapas do processo decisório (estruturação, avaliação e recomendação); e, (vi) a incorporação de uma linguagem que é, ao mesmo tempo, linear (fazendo uso da linguagem humana natural) e não-linear (fazendo uso de representações visuais, que permitem a simultaneidade da informação).

Ainda, no que se refere a atividade de apoio à decisão, Costa (1995) afirmam que a atividade de apoio à decisão pode ser vista como um processo de interação com uma situação problemática mal estruturada onde os elementos e as suas relações emergem de forma mais ou menos caótica.

Já para Roy (1993), a atividade de apoio à decisão é definida como a atividade em que um facilitador, utilizando-se de procedimentos científicos, ajuda a obter elementos de resposta a questões perguntadas aos atores envolvidos em um processo decisório, elementos estes que ajudam a clarificar esta decisão com a finalidade de fornecer aos atores as mais favoráveis condições possíveis para o tipo de comportamento que aumentará a coerência entre a evolução do processo, de um lado, e as metas e/ou sistemas de valores em que esses atores operam, por outro lado (ROY, 1993).

Bouyssou (1990) sintetiza seus pensamentos a respeito da atividade de apoio à decisão da seguinte forma: apoio à decisão consiste em tentar fornecer respostas a perguntas levantadas por atores envolvidos em um processo decisório usando um modelo claramente especificado.

Diante das definições apresentadas acima, pode-se dizer que: (a) A MCDA-C tem como objetivo central possibilitar, aos indivíduos envolvidos em um processo decisório, aumentar o seu grau de conformidade e de entendimento entre a evolução de um processo de tomada de decisão, na presença de seus sistemas de valor e objetivos (pontos de vista); (b) a metodologia inclui o papel do facilitador no processo de ajuda à decisão; e, (c) sua preocupação central é encontrar a solução de melhor compromisso segundo a percepção e valores dos decisores.

Outro ponto a ser salientado é o fato de que a atividade de apoio à decisão não vem por substituir à tomada de decisão em si; ambas são atitudes diferentes e fundamentais neste contexto. $\mathrm{O}$ apoio à decisão, desempenhado pela pessoa do facilitador, não pode ser visto como uma atitude restrita ao favorecimento do entendimento de um problema. Muito pelo contrário, para conseguir possibilitar o entendimento do problema, esta atitude necessita identificar alguns aspectos, freqüentemente caracterizados por dificuldade de evidenciamento, a saber: (a) a identificação do conjunto de ações potenciais (e da indefinição de suas fronteiras); (b) a construção dos critérios; e, (c) a modelação das preferências. Em outras palavras, a atividade de apoio à decisão tem como objetivo fornecer as informações sobre as

RAI - Revista de Administração e Inovação, São Paulo, v. 5, n. 2, p. 150-163, 2008. 
ARTIGOS - Inovação no processo de avaliação do desempenho organizacional: o uso da dimensão integrativa questões que vão surgindo e orientar e apoiar o decisor com relação àquelas que mais atendem as suas expectativas para que tome decisões mais informadas, fundamentadas e claras, em um determinado problema. A tomada de decisão se reveste de uma enorme subjetividade, por ser avaliada segundo os juízos de valor do decisor, a quem compete escolher e decidir.

Neste contexto, conclui-se que a atividade de apoio à decisão se faz fundamentalmente presente em todas as etapas do processo decisório, conforme figura 1. Na fase de estruturação, esta atividade se insere no processo visando a construção de uma estrutura consensualmente aceita pelos atores. Verifica-se, então, que ela não procura modelar uma realidade preexistente, muito pelo contrário. Na fase de avaliação, a atividade de apoio, seguindo uma conduta de interação e aprendizagem, sendo, conseqüentemente, construtivista, desenvolve um modelo no qual as ações potenciais serão avaliadas. Verifica-se, então, que a atividade de apoio não procura seguir um caminho normativo, nem buscar pela solução ótima. Na fase de recomendação, esta atividade procura fornecer subsídios, aos decisores, por meio de algumas ferramentas, para que estes tenham condições de analisar a estratégia mais adequada a ser adotada em cada cenário específico. A seguir, a Figura 1 apresenta as fases do processo decisório sob a perspectiva da MCDA-C.

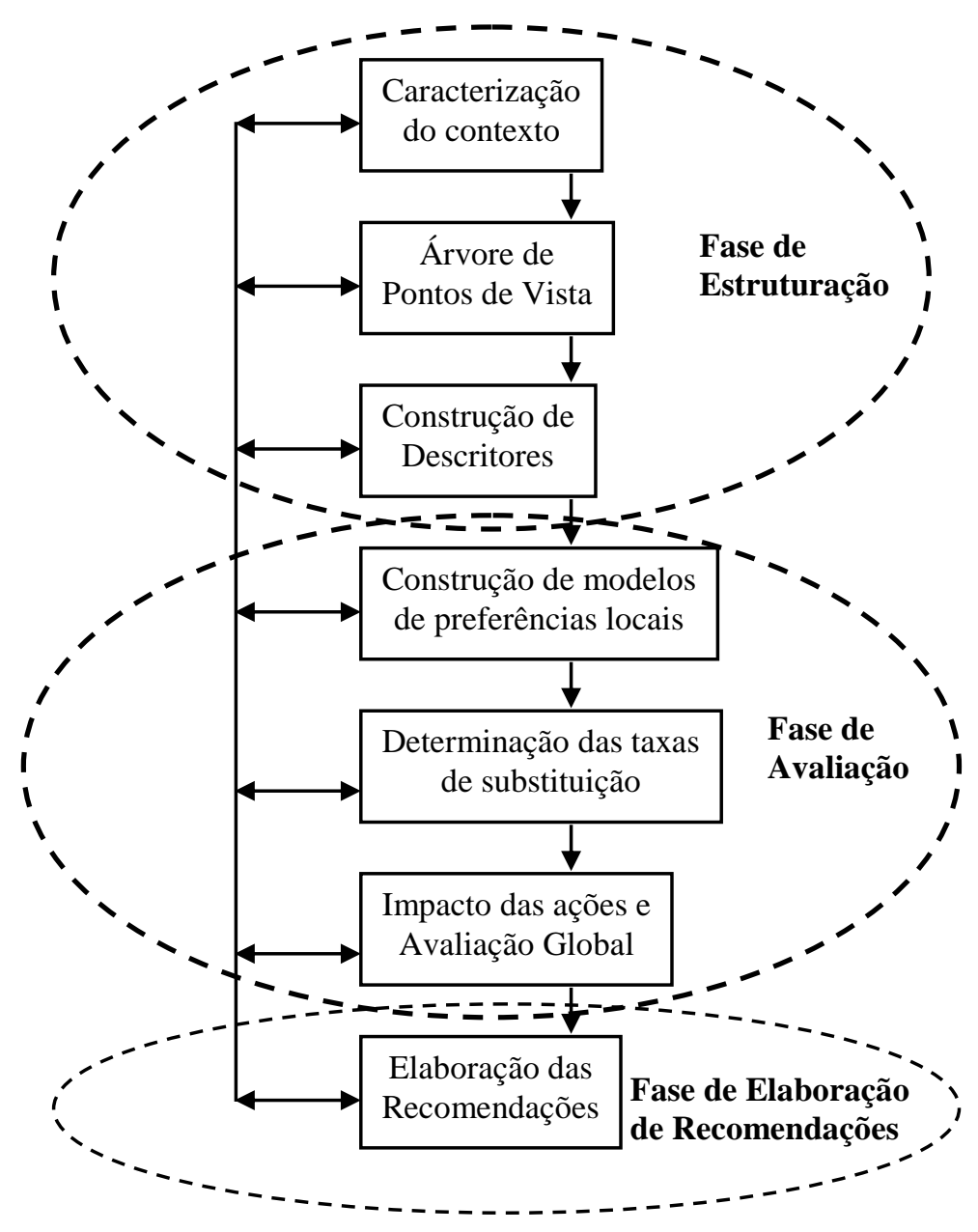

Figura 1 - Processo Decisório sob a perspectiva da MCDA-C

Fonte: Dutra (1998, p. 83)

Diante da visualização permitida pela figura acima, e considerando-se o fato de que a atividade de apoio é feita de forma contínua e interativa, apresentando-se, portanto, como dinâmica e recursiva, pode-se concluir que o processo de tomada de decisão não é 
Ademar Dutra, Leonardo Ensslin, Sandra Rolim Ensslin, Marcus Vinícius Andrade Lima e Ana Lucia Miranda Lopes

linear e seqüencial. Ou seja, a maneira de conduzir este processo deve ser cíclica e dinâmica. Estas características de condução - cíclica e dinâmica - são indicadas, na Figura 1 pelas setas de todas as etapas cruciais e pelas sobreposições das elipses, entre as três fases fundamentais.

Neste sentido, dois aspectos devem ser salientados: (a) o processo de tomada de decisão se caracteriza pela possibilidade de recursividade total, o que não significa, porém, que se pode inverter a ordem de realização das etapas; (b) a elaboração das recomendações, que além de se constituir como uma fase específica, também se constitui ao término do processo de tomada de decisão, como uma etapa interveniente (inserida) em todas as demais, uma vez que o facilitador se faz presente em todos os momentos.

Para cumprir sua função, a metodologia MCDA-C faz uso de três fases, diferenciadas, mas correlacionadas: (i) a estruturação do contexto decisório; (ii) a construção de um modelo de avaliação de alternativas/ações; e, (iii) a formulação de recomendações para os cursos de ações de aperfeiçoamento.

\section{INCORPORAÇÃO DA DIMENSÃO INTEGRATIVA}

O ambiente organizacional é caracterizado por um sistema e seus subsistemas, podendo, ainda, apresentar outros desdobramentos, de acordo com a estrutura da organização ou de seus processos de trabalho. Na perspectiva da avaliação do desempenho organizacional, $\mathrm{o}(\mathrm{s})$ decisor(es) pode(em) estabelecer um parâmetro macro ou micro, observados os diversos níveis hierárquicos presentes na organização. No entanto, as preocupações em termos de desempenho variam em cada nível organizacional, pois as preocupações do decisor de um subsistema organizacional serão diferentes das preocupações de um decisor do sistema organizacional, apesar de todos estes aspectos convergirem para as estratégias e objetivos macros da organização, mantendo a devida coerência e alinhamento.

Na Figura 2 apresenta-se um sistema organizacional com o desdobramento de seus subsistemas.

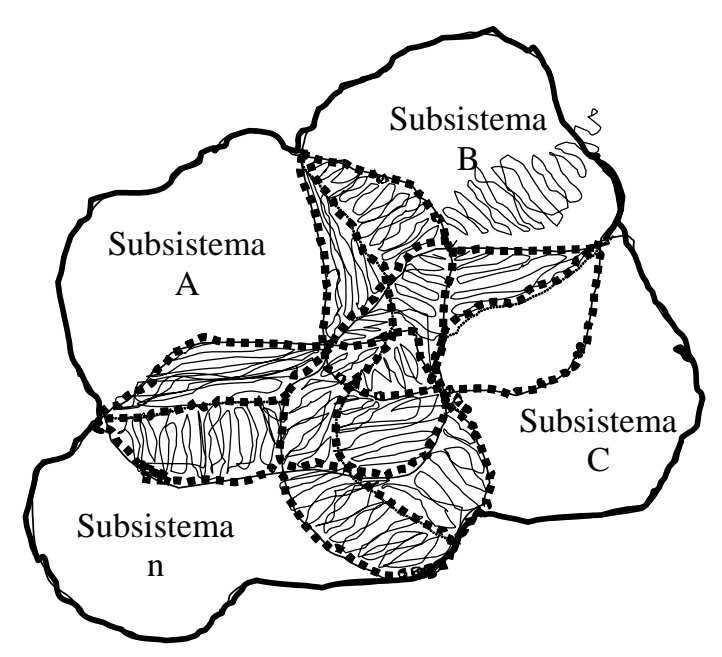

Figura 2 - O Sistema e os Subsistemas Organizacionais

Fonte: Dutra (2003, p. 197)

É no contexto visualizado na Figura 2 que Checkland (1993) explora quatro idéias básicas, que caracterizam um sistema, ou seja: emergência, hierarquia, comunicação e controle, presentes quando aplicadas à atividade humana. Cabe destaque ao primeiro par de idéias (emergência e hierarquia). Checkland (1993) afirma que a propriedade de emergência é decorrente do todo, por meio das contribuições de suas partes, que estão em um nível hierárquico diferente, em relação a este todo.

RAI - Revista de Administração e Inovação, São Paulo, v. 5, n. 2, p. 150-163, 2008. 
ARTIGOS - Inovação no processo de avaliação do desempenho organizacional: o uso da dimensão integrativa

A Figura 2 ajuda a explicitar como ocorre a dimensão integrativa em um processo de avaliação do desempenho organizacional. Observa-se que cada subsistema organizacional mantém inter-relações e interconexões com os demais subsistemas. O ponto de partida para a avaliação do desempenho organizacional, neste contexto, inicia-se com a análise da performance dos subsistemas organizacionais. Assim, o processo de avaliação do desempenho levará em consideração: (i) os aspectos relevantes para o desempenho do subsistema; (ii) os aspectos que um subsistema afeta o desempenho de outro subsistema; (iii) os aspectos que um subsistema é afetado pelo desempenho dos demais subsistemas; e, (iv) a agregação dos desempenhos internos de cada subsistema e das inter-relações e interconexões entre os diversos subsistemas. Destaca-se que este processo ocorre sem perda de foco da convergência com os macro objetivos do sistema organizacional.

Cabe destacar, também, que a metodologia base para o desenvolvimento do presente trabalho é fundamentada no MCDA Construtivista, devidamente apresentada no item 2 deste artigo.

É oportuno esclarecer que os modelos de avaliação de desempenho organizacional, gerados a partir da Metodologia MCDA-C, ajudam a construir o entendimento do decisor em relação à sua percepção da realidade. Tradicionalmente, no âmbito da avaliação do desempenho, a MCDA tem sido utilizada, com sucesso, na formulação de modelos para sistemas organizacionais, limitados à área de comando do decisor, sem a preocupação da integração das partes.

Assim, para fins do objetivo desta pesquisa, ou seja, a incorporação da dimensão integrativa no processo de avaliação de desempenho organizacional, a partir da ferramenta MCDA-C, propõem-se o desenvolvimento das seguintes etapas:

a) Identificação dos subsistemas organizacionais.

Esta etapa visa identificar os subsistemas organizacionais, a partir da estrutura organizacional e ou dos processos de trabalho da organização, como pode ser observado na Figura 3.

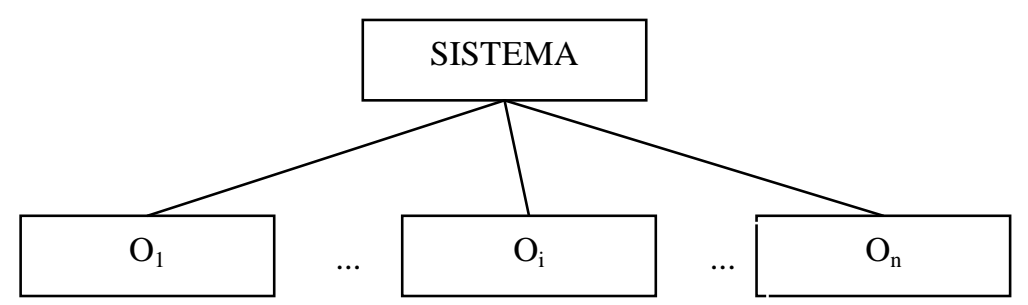

Figura 3 - O Sistema Organizacional e seus Subsistemas

Fonte: Elaborado pelos autores

Pode-se observar na Figura 3, a representação de um sistema organizacional contemplado seus subsistemas $\left(\mathrm{O}_{1}, \mathrm{O}_{\mathrm{i}}, \mathrm{O}_{\mathrm{n}}\right)$.

b) Construção de um modelo de avaliação para cada subsistema, a partir da MCDA-C.

Nesta etapa, deve-se conceber um modelo de avaliação de desempenho para cada subsistema organizacional, a partir da Metodologia MCDA-C. A Figura 4 representa um recorte de um modelo de avaliação de desempenho de um subsistema organizacional.

RAI - Revista de Administração e Inovação, São Paulo, v. 5, n. 2, p. 150-163, 2008. 
Ademar Dutra, Leonardo Ensslin, Sandra Rolim Ensslin, Marcus Vinícius Andrade Lima e Ana Lucia Miranda Lopes

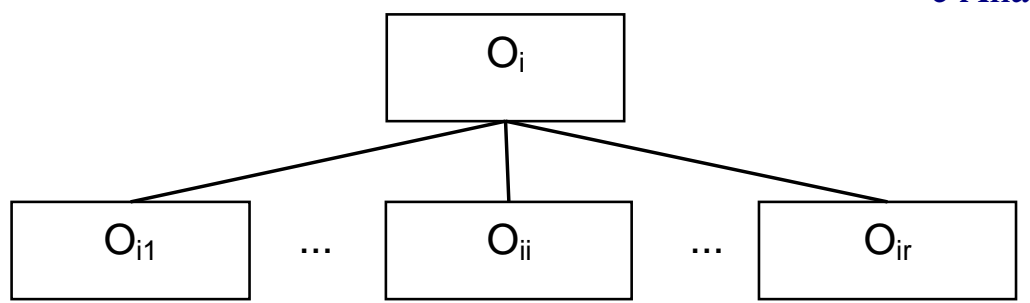

Figura 4 - Representação de Modelo de Avaliação de um Subsitema

Fonte: Dutra (2003, p. 189)

Pode-se observar, na Figura 4 que a representação do modelo de avaliação de um subsistema é composto, no primeiro nível hierárquico $\left(\mathrm{O}_{\mathrm{i})}\right.$ pelo objetivo maior do subsistema, ou seja, avaliar o seu desempenho, e no segundo nível hierárquico $\left(\mathrm{O}_{\mathrm{i} 1}, \mathrm{O}_{\mathrm{ii}}, \mathrm{O}_{\mathrm{ir}}\right)$ pelos seus objetivos estratégicos considerados relevantes pelo decisor. Destaca-se que além do segundo nível hierárquico outros podem ser desmembrados até o estágio passível de medição, ou seja, de construção dos descritores nos termos da MCDA-C, Fase de Estruturação.

c) Identificação das inter-relações e interconexões existente entre os diversos subsistemas no modelo de avaliação previsto no item anterior.

Esta terceira etapa visa a construção de um modelo de avaliação que contemple as inter-relações e interconexões entre os diversos subsistemas, observados:(i) os aspectos em que um subsistema afeta o desempenho de outro subsistema (horizontal de causa); e, (ii) os aspectos em que um subsistema é afetado pelo desempenho de outro subsistema (horizontal de efeito). A Figura 5 apresenta a representação do modelo de avaliação de desempenho de um subsistema organizacional, contemplando a dimensão integrativa.

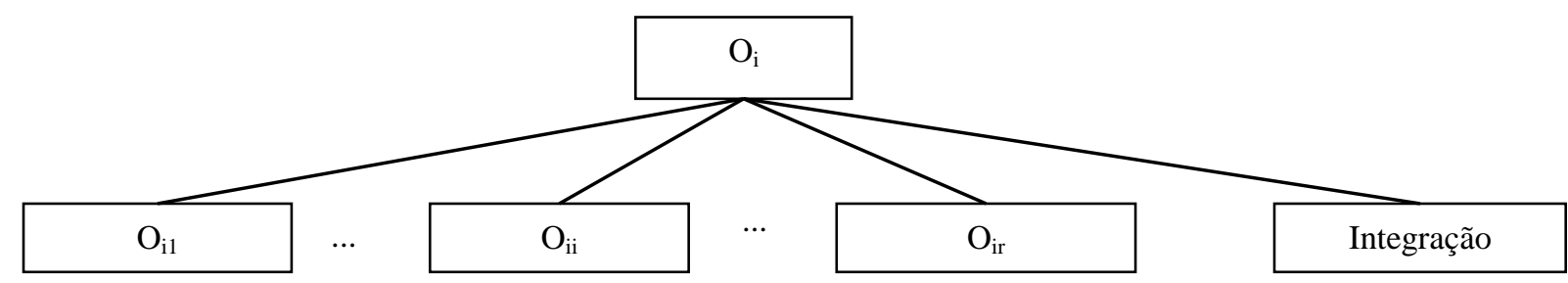

Figura 5 - Representação de um Modelo de Avaliação de um Subsistema contemplando as Inter-Relações com outros Subsistemas

Fonte: Dutra (2003, p. 189)

Pode-se observar, na Figura 5 as variáveis presente na Figura 4, com ênfase na inclusão de um objetivo estratégico denominado integração, que contempla as inter-relações e interconexões com os demais subsistemas. Ou seja, no objetivo estratégico integração são contemplados os aspectos que os outros subsistemas organizacionais afetam o subsistema $\mathrm{O}_{\mathrm{i}} \mathrm{e}$ os aspectos em que o subsistema $\mathrm{O}_{\mathrm{i}}$ afeta o desempenho dos demais subsistemas. Para atender estas especificidades o objetivo estratégico integração precisa ser desmembrado em novos níveis hierárquicos, visando contemplar as variáveis de desempenho especificadas. Entendese que quanto um subsistema afeta o desempenho de outro subsistema ocorre uma relação horizontal de causa. Já quanto um subsistema é afetado pelo desempenho de outro subsistema a relação é horizontal de efeito.

d) Agregação das variáveis de desempenho (alíneas "b" e “c") em um único modelo. 
ARTIGOS - Inovação no processo de avaliação do desempenho organizacional: o uso da dimensão integrativa

Esta quarta etapa visa agregar, em um único modelo, os resultados das variáveis de desempenho de cada subsistema e das inter-relações e interconexões entre os diversos subsistemas, conforme pode-se observar na Figura 6.

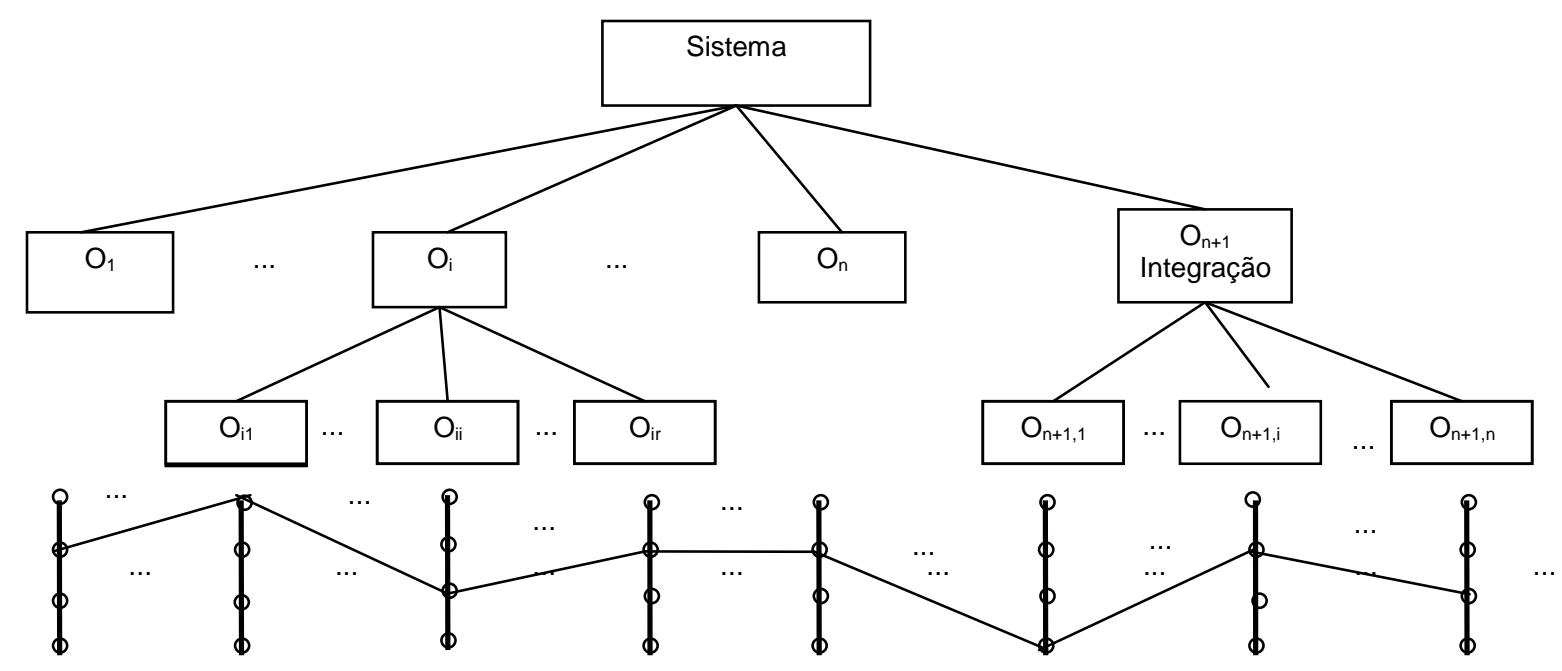

Figura 6 - Representação da Agregação do Modelo de Avaliação, com a Identificação do Perfil de Desempenho

Fonte: Dutra (2003, p. 190)

Constata-se na Figura 6 a representação do sistema organizacional devidamente caracterizado pelos seus subsistemas $\left(\mathrm{O}_{1}, \mathrm{O}_{\mathrm{i}}, \mathrm{O}_{\mathrm{n}}\right)$. No mesmo nível dos subsistemas apresenta-se a área de integração $\left(\mathrm{O}_{\mathrm{n}+1}\right)$, evidenciando a agregação das variáveis de desempenho que um subsistema afeta e é afetado pelo desempenho dos demais subsistemas. Cabe esclarecer que, na Figura 5, a identificação das inter-relações (integração) estava prevista no âmbito do próprio subsistema, isto é, no mesmo nível hierárquico dos objetivos estratégicos de cada subsistema. Já na Figura 6, observa-se que esta área (integração) está prevista no mesmo nível hierárquico dos subsistemas, procedimento este decorrente do processo de agregação. Tal alteração decorre da tendência natural de cada subsistema preocupar-se com seu desempenho interno, ficando, em segundo plano as contribuições para que os outros subsistemas tenham um melhor desempenho (inter-relações). Destaca-se ainda na parte inferior da representação acima (Figura 6) o perfil de desempenho do sistema organizacional, obtido a partir da construção de descritores para cada subsistema, nos termos da Metodologia MCDA-C, conforme apresentado no item 2 deste artigo, assim como o processo de agregação aditiva do modelo, que resulta na performance global de desempenho do sistema.

Ainda, a partir da Figura 6, observa-se que um subsistema possui variáveis de desempenho internas, isto é, exclusivas de sua área de atuação e aspectos inter-relacionados com os demais subsistemas do ambiente organizacional, caracterizado pela área $\mathbf{O}_{\mathbf{n}+1}$. Neste caso, um subsistema pode afetar o desempenho de outro subsistema ou ser por ele afetado. Este processo de inter-relação, isto é, de influenciar ou ser influenciado, torna-se cada vez mais freqüente no ambiente organizacional, dada à complexidade deste ambiente, a integração dos processos de trabalho e a competitividade das organizações.

e) Identificação das estratégias mais apropriadas para o sistema como um todo.

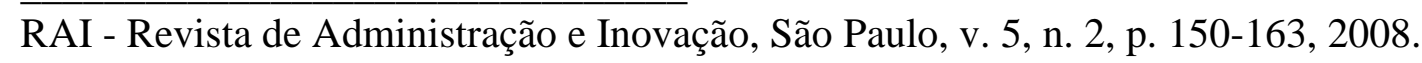


Ademar Dutra, Leonardo Ensslin, Sandra Rolim Ensslin, Marcus Vinícius Andrade Lima e Ana Lucia Miranda Lopes

Esta quinta etapa visa, a partir do perfil de desempenho de cada subsistema e do sistema organizacional como um todo, identificar as estratégias de aperfeiçoamento mais adequadas para o sistema. Com isso, permite-se de forma transparente, descobrir quais escolhas estratégicas, considerando-se as variáveis de desempenho (internas e interrelacionais) de cada subsistema, são mais adequadas para um melhor desempenho do sistema como um todo, possibilitando um equilíbrio dinâmico e global.

Neste sentido, o sistema organizacional pode fazer valer sua hierarquia e, mesmo delegando autonomia para cada subsistema atuar da melhor maneira possível no contexto interno, controlar e supervisionar suas inter-relações, visando orientar os esforços, prioritariamente, para o alcance dos objetivos do todo. Assim, cada parte (subsistema) demandará mais energia nos aspectos que terão maior impacto no resultado global do sistema. Exemplificando, cada subsistema, à medida em que se deparar com duas alternativas pontuais - ou aperfeiçoar o seu desempenho interno em determinado aspecto, ou contribuir para que outro subsistema tenha um melhor desempenho - deverá ser orientado para optar pela alternativa que apresentar maior contribuição para o desempenho global do sistema.

Sintetizando, a incorporação da dimensão integrativa no processo de avaliação de desempenho organizacional tem como foco a busca da melhor forma para se obter o máximo desempenho global do sistema organizacional, onde se potencializa a participação de cada parte (subsistema) na melhoria das variáveis de seu desempenho interno e/ou, principalmente na ênfase das inter-relações. Assim, cada parte constituinte estará focada na alavancagem do melhor rendimento global do sistema, tornando-o superior a soma das partes.

Com isso, constata-se a potencialidade da dimensão integrativa, associada à Metodologia MCDA Construtivista, no processo de avaliação de desempenho organizacional. Tal potencialidade se explica pela agregação cumulativa de variáveis, culminando na possibilidade de consideração do equilíbrio dinâmico e global do sistema. Cabe esclarecer que o equilíbrio dinâmico e global do sistema organizacional se dá pela conjunção de esforços, na escolha de estratégias mais apropriadas para o sistema como um todo.

Destaca-se ainda, que na visão de sistemas fechados como proposta por Maturana e Varela (1997) - os sistemas, para evoluírem, devem ter a capacidade de, além de desempenharem suas atividades funcionais de maneira competitiva, devem intercambiar energia com o exterior em uma forma endotérmica. Adicionalmente, esses autores afirmam que este intercâmbio deve ser regulado pelos interesses do sistema maior, o que, igualmente, está contemplado na MCDA-C incorporada a dimensão integrativa, quando propõe a avaliação e gestão do processo de intercâmbio por meio da figura hierárquica superior (sistema maior), sem tolher a liberdade e iniciativa dos subsistemas, tanto no que se refere ao desempenho de suas atividades internas, quanto à forma de alcançar o desempenho de seus inter-relacionamentos.

No contexto das premissas apresentadas, cita-se, como metáfora para melhor ilustrar a dimensão integrativa, a relação entre um casal (marido e esposa). Considerando-se esta relação como um sistema, é possível identificar os objetivos e desejos do casal; considerando-se cada membro do casal com um subsistema, é possível identificar os objetivos e desejos de cada parte, marido ou esposa. A Figura 7 é uma representação do sistema casal, onde são constatados os objetivos de cada parte e uma área que contempla os objetivos interrelacionados.

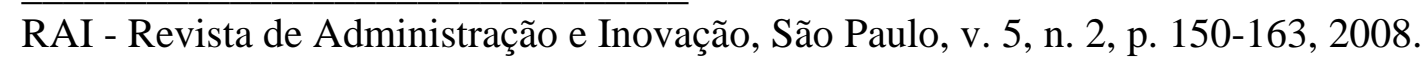


ARTIGOS - Inovação no processo de avaliação do desempenho organizacional: o uso da

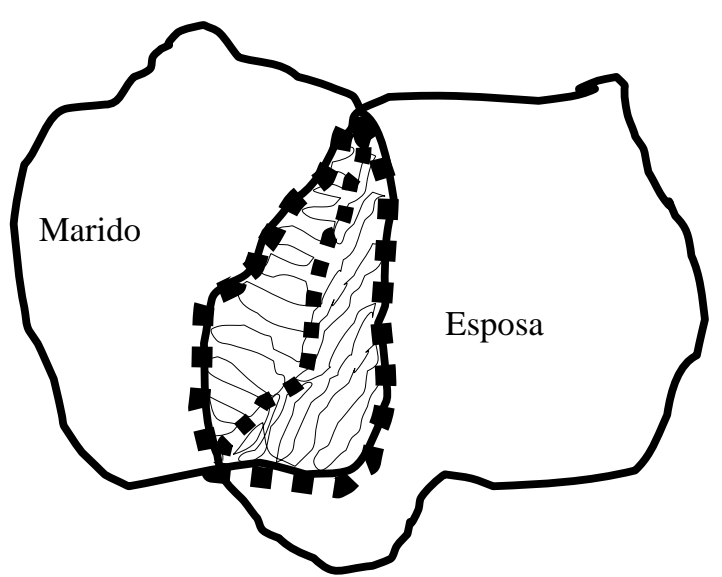
dimensão integrativa

Figura 7 - Sistema Casal Constituído pelos Subsistemas Marido e Subsistema Esposa Fonte: Dutra (2003, p. 194)

Observa-se na Figura 7, que a organização (sistema casal) é constituída por: (i) um conjunto de objetivos específicos ao marido; (ii) um conjunto de objetivos específicos à esposa; e, (iii) um conjunto de objetivos mútuos (inter-relacionais).

Correlacionando ao contexto organizacional pode-se afirmar que as variáveis marido e esposa equivalem aos subsistemas, conforme representado na Figura 8.

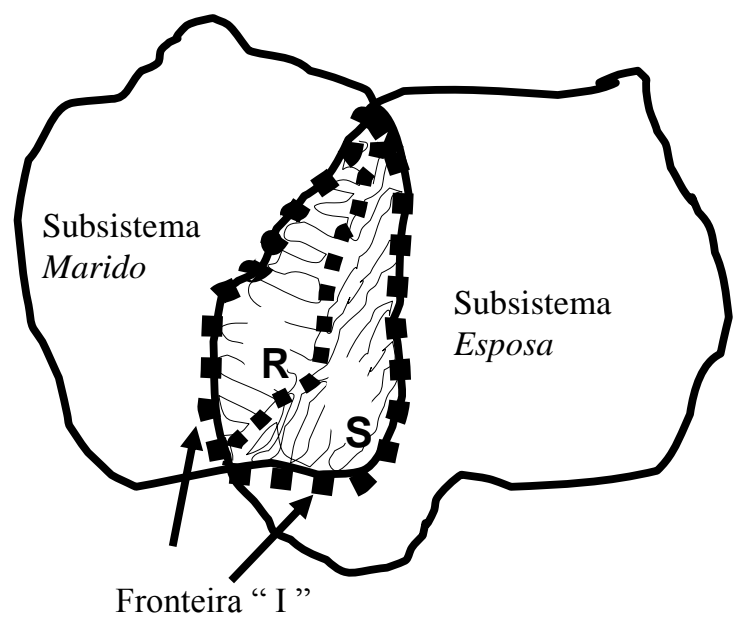

Figura 8 - Subsistemas Marido e Esposa e seus Inter-Relacionamentos

Fonte: Dutra (2003, p. 197)

Constata-se, a existência dos subsistemas marido e esposa, com a identificação da área de inter-relacionamento, onde a área " $R$ " identifica que o subsistema esposa afeta $o$ desempenho do subsistema marido e a área "S" identifica que o subsistema marido afeta o desempenho do subsistema esposa. A fronteira "I", que delimita o desempenho interno de cada subsistema, é móvel, sendo sua mobilidade resultado do quão comprometido cada subsistema estiver com o desempenho do outro e, por consequiência, da união do casal (sistema) como um todo.

Assim, o sistema casal poderá ter, então, seu desempenho medido utilizando-se da Metodologia MCDA Construtivista, conforme modelo ilustrado na Figura 9. Destaca-se que, nesta ilustração, a noção de desempenho é equacionada através do rótulo Felicidade do Casal. 


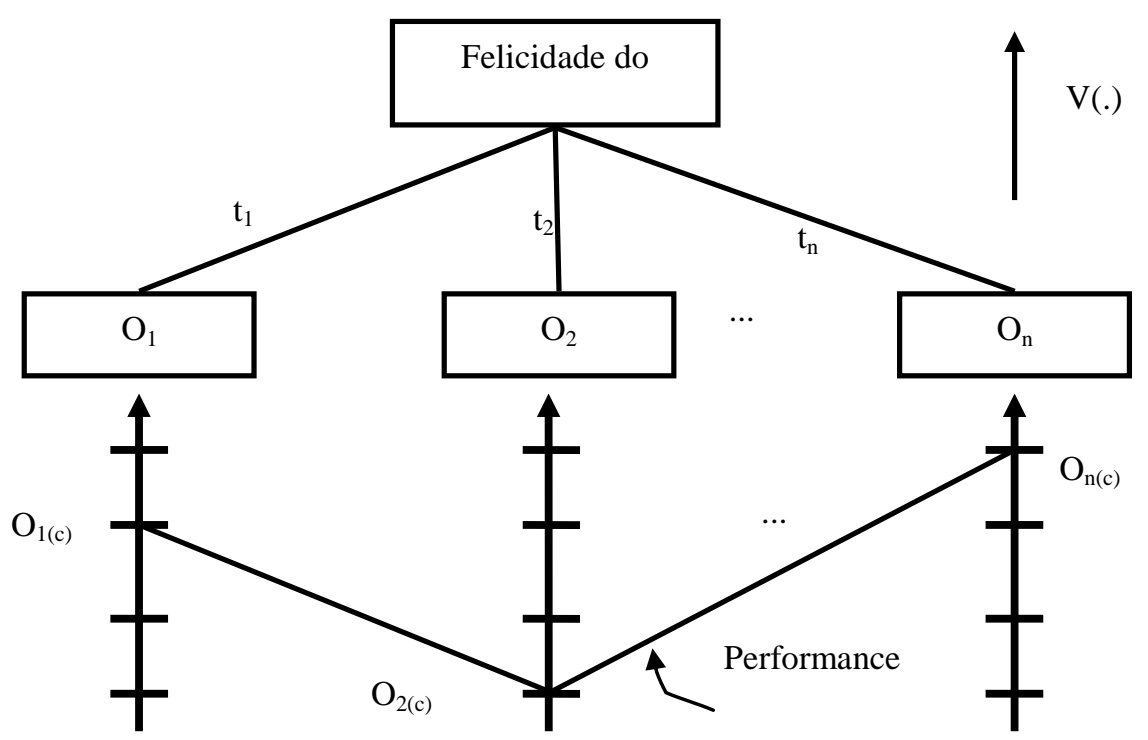

Figura 9 - Modelo de Avaliação do Sistema Casal "C"

Fonte: Dutra (2003, p. 195)

Como pode ser observado na

Figura 9, a Felicidade do Casal é obtida pelo grau de realização (alcance) de seus objetivos (objetivos do marido $-\mathrm{O}_{1}$, objetivos da esposa $-\mathrm{O}_{2}$, objetivos mútuos - $\mathrm{O}_{\mathrm{n}}$ ), possibilitando uma avaliação local e global, por meio do processo de agregação aditiva.

No contexto desta ilustração, a visão proposta pela MCDA Construtivista, com a incorporação da dimensão integrativa, diz respeito à organização ou sistema maior faz valer sua hierarquia e, mesmo delegando autonomia para cada subsistema realizar suas funções internas, supervisiona as inter-relações visando orientar os esforços, prioritariamente, para a potencialização do alcance dos objetivos do todo.

No caso da organização ou sistema Casal, cada um dos subsistemas marido e esposa têm autonomia para buscar o melhor desempenho em seus objetivos independentes, bem como na forma de ajudar o outro subsistema, desde que, prioritariamente, maximizem o desempenho do todo.

Assim, a incorporação da dimensão integrativa, como proposta neste artigo, visa identificar esta área de inter-relacionamento, promovendo a integração e buscando formas de melhoria de desempenho, sem, no entanto, abrir mão do desempenho independente de cada subsistema, desde que, simultaneamente, atenda o balanceamento (interrelacionamentos) que irá maximizar os resultados do todo.

\section{CONSIDERAÇÕES FINAIS}

No decorrer deste artigo, pode-se constatar a potencialidade da dimensão integrativa, associada à Metodologia MCDA Construtivista - MCDA-C, tornando-se uma importante inovação no processo de avaliação de desempenho organizacional. Tal potencialidade se explica pela agregação cumulativa de variáveis (dos subsistemas para o sistema organizacional), culminando na possibilidade de consideração do equilíbrio dinâmico e global do sistema. Cabe esclarecer que o equilíbrio dinâmico e global do sistema organizacional se dá pela conjunção de esforços, na escolha de estratégias mais apropriadas para o sistema como um todo.

A incorporação da dimensão integrativa objetiva demonstrar que é possível avaliar o desempenho de uma organização, a partir de seus subsistemas, permitindo alavancar 
ARTIGOS - Inovação no processo de avaliação do desempenho organizacional: o uso da dimensão integrativa a melhoria do desempenho, a partir da escolha de estratégias que melhor potencializem a performance do sistema organizacional como um todo.

$\mathrm{O}$ referencial metodológico apresentado identifica as etapas a serem seguidas para a incorporação da dimensão integrativa, permitindo observar a potencialidade de ganhos organizacionais com tal prática.

No contexto organizacional, a incorporação da dimensão integrativa, permite o alcance dos seguintes resultados: (i) identificar as variáveis que um subsistema afeta e é afetado pelo desempenho de outro subsistema; (ii) dispor de informações para orientar que cada subsistema, à medida em que se deparar com duas alternativas pontuais - ou aperfeiçoar o seu desempenho interno em determinado aspecto, ou contribuir para que outro subsistema tenha um melhor desempenho - opte pela alternativa que apresentar maior contribuição para o desempenho global do sistema; (iii) identificar quais escolhas estratégicas são mais adequadas, para um melhor desempenho do sistema como um todo, permitindo um equilíbrio dinâmico e global; e, (iv) estimular e garantir um processo de comunicação constante entre os diversos subsistemas e o sistema organizacional como um todo.

Sintetizando, a incorporação da dimensão integrativa no processo de avaliação de desempenho organizacional tem como foco a busca da melhor forma para se obter o máximo desempenho global do sistema organizacional, onde se potencializa a participação de cada parte (subsistema) na melhoria das variáveis de seu desempenho interno e/ou, principalmente, na ênfase nas inter-relações e interconexões. Assim, cada parte constituinte estará focada na alavancagem do melhor rendimento global do sistema, tornando-o superior à soma das partes.

Cumpre observar que a identificação dos efeitos advindos da incorporação da dimensão integrativa na sua totalidade é complexa e explica uma das limitações de sua aplicabilidade, sobretudo no que diz respeito à resistência das organizações em adotar uma perspectiva sistêmica em seus processos de gestão.

Apesar disto, reconhece-se, entretanto, a contribuição que a dimensão integrativa pode trazer ao processo de avaliação de desempenho organizacional. $O$ que se propõe, nesta pesquisa é evidenciar as vantagens da exploração da nova dimensão, bem como oferecer procedimentos metodológicos de sua implementação, para, como etapa final, possibilitar a melhoria do processo de avaliação de desempenho organizacional.

\section{REFERÊNCIAS}

ALAVI, M.; CARLSON, P. A review of MIS research and disciplinary development. Journal of Management Information Systems, Armonk, v. 8, n. 4, p. 45-62, 1992.

BOUYSSOU, D. Building Criteria: a prerequisite for MCDA. In: Bana e Costa, C. A. (Ed.)

Readings in Multiple Criteria Decision Aid. Berlin: Springer, p.58-82, 1990.

CHECKLAND, P. Systems thinking, systems practice. Chichester: John Wiley \& Sons, 1993.

COSTA, C. A. B. Introdução geral às abordagens multicritério de apoio à tomada de decisão. Investigação Operacional, Lisboa, v. 8, n. 1, p. 117-139, Jun. 1988.

COSTA, C. A. B. Processo de apoio à decisão: problemáticas, actores e acções. Florianópolis: ENE/UFSC, 1995

COSTA, C. A. B. Readings in multiple criteria decision aid. Berlim: Springer-Verlag, 1990.

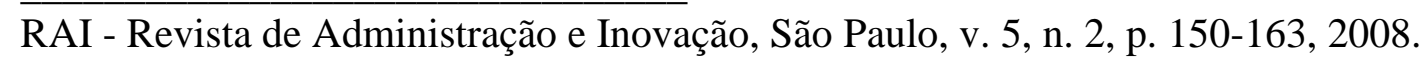


Ademar Dutra, Leonardo Ensslin, Sandra Rolim Ensslin, Marcus Vinícius Andrade Lima e Ana Lucia Miranda Lopes COSTA, C. A. B. Três convicções fundamentais na prática do apoio à decisão. Pesquisa Operacional, Rio de Janeiro, v. 13, n. 1, p. 9-20, Jun. 1993.

COSTA, C. A. B.; VINCKE, P. Multiple criteria decision aid: an overview. In: COSTA, C. A. B. (Ed.). Readings in multiple criteria decision aid. Berlin: Springer-Verlag, 1990. p. 3-14.

DUTRA, A. Elaboração de um sistema de avaliação de desempenho dos recursos humanos da Secretaria de Estado da Administração - SEA à luz da metodologia multicritério de apoio à decisão 1998. 443 f. Dissertação (Mestrado) - Programa de PósGraduação em Engenharia de Produção, Universidade Federal de Santa Cataria, Florianópolis, 1998.

DUTRA, A. Metodologia para avaliar e aperfeiçoar o desempenho organizacional: incorporando a dimensão integrativa à MCDA construtivista-sistêmico-sinergética. 2003. 320 f. Tese (Doutorado) - Programa de Pós-Graduação em Engenharia de Produção, Universidade Federal de Santa Cataria, Florianópolis, 2003.

ENSSLIN, L.; MONTIBELlER NETO, G.; NORONHA S. M. Apoio à decisão. Florianópolis: Insular; 2001.

ENSSLIN, S. R.; LIMA, M. V. A. Apoio à tomada de decisão estratégica: uma proposta metodológica construtivista. In: ANGELONI, M. T.; MUSSI, C. C. (Org.). Estratégias: formulação, implementação e avaliação - o desafio das organizações contemporâneas. São Paulo: Saraiva, 2008. p. 243-270.

IGARASHI, D. C. C. et al. A qualidade do ensino sob o viés da avaliação de um programa de pós-graduação em contabilidade: proposta de estruturação de um modelo híbrido: proposta de estruturação de um modelo híbrido. Revista de Administração, São Paulo, v. 43, n. 2, p. p.117-137, abr./maio/jun. 2008.

KEENEY, R. L. Value focused-thinking: a path to creative decision-making. Cambridge: Harvard University Press, 1992.

LANDRY, M. A note of the concept of 'problem'. Organization Studies, Berlin, v. 16, n. 2, p. 315-343, 1995.

MATURANA, H. R.; VARELA, F. J. Autopoiesis and cognition: the realization of the living. Dordrecht: D. Reidel Publishing Company, 1997. (Boston Studies in the Philosophy of Science, v. 42).

NCKE, P. Multicriteria decision-aid. Chichester: John Wiley \& Sons, 1990.

RICHARDSON, R. J. Pesquisa social: métodos e técnicas. 3. ed. São Paulo: Atlas, 1999.

ROY, B. Decision science or decision-aid science? European Journal of Operational Research, Amsterdam, v. 66, n. 2, p. 184-203, Apr. 1993.

ROY, B.; VANDERPOOTEN, D. The European school of MCDA: emergence, basic features and current works. Journal of Multi-Criteria Decision Analysis, Glasgow, v. 5, n.1, p. 2238, Mar. 1996.

\section{INNOVATION IN THE PROCESS OF ORGANIZATION PERFORMANCE EVALUATION: THE USE OF THE INTEGRATIVE DIMENSION}

\section{Abstract}

RAI - Revista de Administração e Inovação, São Paulo, v. 5, n. 2, p. 150-163, 2008. 
ARTIGOS - Inovação no processo de avaliação do desempenho organizacional: o uso da dimensão integrativa

The objective of this article is to discuss the incorporation of the integrative dimension in the process of organization performance evaluation by drawing upon the Methodology Multicriteria Decision-Aid Constructivist - MCDA-C . This dimension seeks to identify the internal performance variables for each organization subsystem, mainly those deriving from the inter-relations and inter-connections among the various subsystems, with an emphasis on the strategic choices which are most relevant to the performance improvement of the system as a whole. As a result, the incorporation of the integrative dimension will: (i) identify the variables which a subsystem affects and those affected by the subsystem in which it is affected by the performance of another subsystem; (ii) allow for each subsystem to choose, between two punctual alternatives for performance improvement, that which will contribute the most to the system global performance; (iii) identify those strategies which are most adequate to improve the performance of the system as a whole thus allowing for a dynamic and global balance.

Keywords: Evaluation, integrative dimension, MCDA-C, organizational performance Eealuation.

Data do recebimento do artigo: 10/06/2008

Data do aceite de publicação: 10/07/2008

RAI - Revista de Administração e Inovaçãa, São Paulo, v. 5, n. 2, p. 150-163, 2008. 